\title{
Electrochemical sensing of methyl parathion on magnetic molecularly imprinted polymer
}

\author{
Amal H.A. Hassan ${ }^{\mathrm{a}, \mathrm{b}}$, Silio Lima Moura ${ }^{\mathrm{a}}$, Fatma H.M. Ali ${ }^{\mathrm{b}}$, Walaa A. Moselhy, \\ Maria del Pilar Taboada Sotomayor ${ }^{\mathrm{d}}$, Maria Isabel Pividori ${ }^{\mathrm{a}, *}$ \\ a Grup de Sensors i Biosensors, Department de Química, Universitat Autònoma de Barcelona (UAB), Bellaterra, 08193 Barcelona, Spain \\ ${ }^{\mathrm{b}}$ Food Hygiene and Control department, Faculty of Veterinary Medicine, Beni-Suef University, 62511 Beni-Suef, Egypt \\ ${ }^{\mathrm{c}}$ Forensic Medicine and Toxicology department, Faculty of Veterinary Medicine, Beni-Suef University, 62511 Beni-Suef, Egypt \\ d Department of Analytical Chemistry, Institute of Chemistry, State University of São Paulo (UNESP), 14801-970 Araraquara, SP, Brazil
}

\section{A R T I C L E I N F O}

\section{Keywords:}

Magnetic molecularly imprinted polymer

Methyl parathion

Fish

Food safety

Electrochemical sensing

\begin{abstract}
A B S T R A C T
The electrochemical detection of methyl parathion in fish was performed by preconcentrating the pesticide on magnetic molecularly imprinted polymer and further readout on magneto-actuated electrode by square wave voltammetry. The magnetic molecularly imprinted polymer was synthesized by a magnetic core-shell strategy, using methacrylic acid as a functional monomer, and selected by theoretical calculation using the density functional theory (DFT). The characterization of this material was performed by SEM, TEM and XRD. Moreover, the binding capacity and selectivity towards methyl parathion was studied and compared with the corresponding magnetic non-imprinted polymer. The magneto-actuated electrochemical sensor showed outstanding analytical performance for the detection of methyl parathion in fish, with a limit of detection of as low as $1.22 \times 10^{-6}$ $\mathrm{mg} \mathrm{L}^{-1}$ and recovery values ranging from $89.4 \%$ to $94.7 \%$. The magnetic molecularly imprinted polymer successfully preconcentrated the analyte from the complex samples and paves the way to incorporate this material in other platforms for the detection of this pesticide in the field of environmental control and food safety.
\end{abstract}

\section{Introduction}

Fish is one of the most popular foods constituting an exceptionally valuable source of protein and omega 3 in the human diet. Unfortunately, fish are exposed to contamination with different types of chemical hazards due to environmental pollution, including pesticides (Vogt et al., 2012). Methyl parathion is a widely used and highly toxic organophosphorus pesticide (Silva et al., 2004), which tends to accumulate in water, soil, and fish due to its low solubility and bioaccumulation properties causing serious health and environmental issues (Lagaly, 2001). It inhibits the cholinesterase enzyme leading to dizziness, headaches, vomiting and in same instances death (Liu and Lin, 2005). Ingestion of contaminated fish with methyl parathion is considered one of the most important routes for human toxicity. As a consequence, fish should be accurately and continuously examined. Continuous monitoring of methyl parathion residue in fish muscles required highly sophisticated, time consuming procedures using benchtop instruments, such as high performance liquid chromatography, gas chromatography, immunoassay and mass spectrometry that usually require sample pretreatment (Shanker et al., 2001; Huang et al.,
2002; Tomkins and Ilgner, 2002; Sanz et al., 2004). Therefore, there is a need for the development of novel alternative methods for in-field analysis of this pesticide and to screen-out food samples in other settings different that the laboratory facilities. Molecularly Imprinted Polymers (MIPs) are synthetic biomimetic materials mimicking biological receptors (Vlatakis et al., 1993). They are highly cross-linked macromolecular structures towards the template which is then extracted after polymerization, originating binding sites towards the template molecule. Although MIPs have in general lower affinity and specificity than the biological counterparts, they show important technological features, including affordable animal-free and large-scale production. Moreover, they show high chemical stability. Since the early reports on magnetic separation technology (Rembaum et al., 1982), magnetic particles (MPs) have been used for preconcentration of a huge range of targets in a variety of applications. They can be easily modified with antibodies for the immunomagnetic separation and preconcentration of different target from complex specimens (Carinelli et al., 2015; Brandão et al., 2015). Furthermore, MPs can be easily incorporated on magneto-electrodes for electrochemical readout using permanent magnets in near contact (Zacco et al., 2006). However, the

\footnotetext{
* Corresponding author.

E-mail address: isabel.pividori@uab.cat (M.I. Pividori).
} 
main drawback of antibody-modified MPs is their high cost and low stability. This fact can be easily solved by merging the outstanding properties of magnetic particles and MIPs, acting as plastic antibodies. All these advantages placed magnetic-MIP as material of choice for many applications in separation techniques and sensors development (Duan et al., 2015; Ma and Shi, 2015). Firstly, this work addresses the rational design of a magnetic molecularly imprinted polymer for the selective recognition of methyl parathion. The selection of the functional monomer was performed among 10 candidates by theoretical calculation of the formation energies of complexes using the density functional theory (DFT) with the program package Gaussian 09. The core-shell synthesis of the magnetic-MIP was thus performed with methacrylic acid as a functional monomer, EGDMA as cross-linking monomer and AIBN as radical initiator. This molecularly-imprinted polymer with magnetic properties (magnetic-MIP) and affinity towards methyl parathion showed an outstanding preconcentration capacity for this pesticide from complex samples such as fish. The quantification of the pesticide was then performed by magnetic-actuation of the magnetic-MIPs for further electrochemical detection, based on the cathodic process of methyl parathion in close proximity to the electrode surface.

\section{Materials and methods}

\subsection{Chemicals and solutions}

All reagents used in the synthesis of magnetic molecularly imprinted polymer (magnetic-MIP) for methyl parathion were analytical grade and purchased from Sigma-Aldrich, while 2,2-Azobis-isobutyronitrile (AIBN) from Fisher Scientific. Methyl parathion stock solutions were prepared with ethanol and stored in a refrigerator at $4{ }^{\circ} \mathrm{C}$. Malathion, 4nitrophenol and phenol were purchased from Sigma-Aldrich and the stock solutions were prepared with ethanol. Britton-Robinson buffer (BR) was $0.04 \mathrm{~mol} \mathrm{~L}^{-1} \mathrm{H}_{3} \mathrm{BO}_{3}, 0.04 \mathrm{~mol} \mathrm{~L}^{-1} \mathrm{H}_{3} \mathrm{PO}_{4}$ and $0.04 \mathrm{~mol} \mathrm{~L}^{-1}$ $\mathrm{CH}_{3} \mathrm{COOH} \mathrm{pH}=2$.

\subsection{Equipment}

Cyclic voltammetry and square wave voltammetry were performed on a $\mathrm{CHI} 650 \mathrm{C}$ electrochemical workstation (CH Instruments, USA) using $\mathrm{CHI} 16.02$ for instrument control and data acquisition. A standard one-compartment three electrodes cell was used, with a $\mathrm{Ag} / \mathrm{AgCl}$ as reference electrode, a platinum wire as counter electrode and a magneto-electrode based on graphite-epoxy composite (m-GEC) as the working electrode (Pividori, Alegret, 2005). The preparation of the electrodes are detailed described in Supp. Data and Fig. S1. Further characterization of the magneto electrodes including reproducibility of the construction, renewal and reusability, and stability has been extensively reported by our research group (Ben Aissa et al., 2017).

UV spectrophotometry measurements were performed in 96-Well Polystyrene UV flat bottom microtiter plates $\left(\mathrm{N}^{\circ} 8404\right.$, Thermofisher, US) using a microplate reader (Infinit 200 PRO, Tecan, Austria) and Magellan 9.0 software for data acquisition and analysis (Tecan, Switzerland). The scanning electron microscopy (SEM) images were taken by electron microscope EVO MA-10 with Energy-dispersive X-ray spectroscopy (EDS) Detector (Oxford LINCA). The transmission electron microscopy (TEM) images were taken by the transmission electron microscope JEM-2011 (with EDS Detector oxford LINCA). Magnetic separation during the washing steps was performed by a magnetic separator Dynal MPC-S (Product N 120.20D, Dynal Biotech ASA, Norway).

\subsection{Computer simulation for the rational design of the molecularly imprinted polymer for methyl parathion}

The theoretical study for the rational design of molecularly imprinted polymer (MIP) for the binding of methyl parathion as a template was performed by density functional theory (DFT). The M062X/6-31G(d,p) level of theory was used to assess the geometry optimization of the monomers-template interactions, also considering the solvation energy (Moura et al., 2018). More details are provided in Supp. data.

\subsection{Synthesis and characterization of the magnetic molecularly imprinted polymers for methyl parathion}

The magnetic-MIP was synthesized following a core-shell procedure, as previously reported by our research group, with minor changes. The details are provided in Supp. data and schematically shown in Fig. S2. Briefly, the synthesis of the magnetite nanoparticles core $\left(\mathrm{Fe}_{3} \mathrm{O}_{4}\right)$ was performed by co-precipitation of a $\mathrm{Fe}^{2+} / \mathrm{Fe}^{3+}$ mixed solution. The $\mathrm{Fe}_{3} \mathrm{O}_{4}$ nanoparticles were modified with tetraethyl orthosilicate (TEOS) to provide $\mathrm{OH}$ groups for further reaction. The following step was the reaction of the hydroxyl modified magnetic particles $\left(\mathrm{Fe}_{3} \mathrm{O}_{4} @ \mathrm{SiO}_{2}\right)$ with the acrylic group containing in the silanizating agent 3-methacryloxypropyltrimethyloxysilane (MPS), to provide activated $C=C$ groups for further co-polymerization in anhydrous toluene, as predicted by the theoretical calculation. The pre-polymerization mixture was performed by mixing the monomer methacrylic acid and the template methyl parathion in a 5:1 stoichiometric ratio in toluene, as predicted by our theoretical calculations. The $\mathrm{Fe}_{3} \mathrm{O}_{4} @ \mathrm{SiO}_{2}$-MPS was added to the prepolymerization mixture with the crosslinking monomer, (ethylene glycol dimetacrylate, EGDMA) and the radical initiator, (azobisisobutyronitrile, AIBN) using toluene as a porogenic solvent. The methyl parathion extraction was done using a Soxhlet and methanol:acetic acid 9:1 as extracting solvent. The solution was changed every $12 \mathrm{~h}$ during five days to ensure the complete removal of methyl parathion. After that, the product was dried at room temperature. Similarly, the magnetic non-molecularly imprinted polymer (magnetic-NIP) was prepared without the presence of the template.

After each step of the core-shell synthesis, the product was collected and characterized by SEM and TEM, as detailed in Supp. data. Energy dispersive X-ray spectroscopy detector (EDS) was also used for the elemental analysis. Other characterization of the material during the synthesis was performed, including selected-area diffraction analysis (SAED), X-ray diffraction, and UV spectrophotometry.

\subsection{Binding and selective adsorption study}

The binding experiments were carried out at room temperature by mixing $5.0 \mathrm{mg}$ of magnetic-MIP or magnetic-NIP with $1.0 \mathrm{~mL}$ of methyl parathion standard solutions of different concentrations ranging from 0.0 to $263.2 \mathrm{mg} \mathrm{L}^{-1}$. The mixture was shaken in a rotary shaker for $60 \mathrm{~min}$. The magnetic polymer suspensions were separated by external neodymium magnetic bar. The absorbance of the supernatant was measured at a wavelength of $270 \mathrm{~nm}$. The amount of methyl parathion adsorbed by magnetic-MIP $\left(Q_{e}, \mathrm{mg} \mathrm{g}^{-1}\right)$ was calculated as follow:

$Q_{e}=\frac{V\left(C_{0}-C_{e}\right)}{m}$

where $Q_{e}\left(\mathrm{mg} \mathrm{g}^{-1}\right)$ is the experimental equilibrium adsorption capacity, $\mathrm{C}_{0}\left(\mathrm{mg} \mathrm{L}^{-1}\right)$ is the initial concentration of methyl parathion, $\mathrm{C}_{\mathrm{e}}\left(\mathrm{mg} \mathrm{L}^{-1}\right)$ is the equilibrium concentration, $\mathrm{V}(\mathrm{mL})$ is the volume of the solution and $\mathrm{m}(\mathrm{mg})$ is the weight of magnetic-MIP or magnetic-NIP.

The effect of binding time was determined by mixing $5.0 \mathrm{mg}$ of magnetic-MIP (and magnetic-NIP) with $1.0 \mathrm{~mL}$ of $26.3 \mathrm{mg} \mathrm{L}^{-1}$ methyl parathion solution and incubated by changing the binding time from 0 to $60 \mathrm{~min}$.

For the study of the influence of magnetic-MIP or magnetic-NIP on the adsorption capacity, different amount of magnetic-MIP (and 
magnetic-NIP) ranging from 1.0 to $5.0 \mathrm{mg}$ were mixed with $1.0 \mathrm{~mL}$ of $26.3 \mathrm{mg} \mathrm{L}^{-1}$ of methyl parathion and incubated for $60 \mathrm{~min}$. In all cases, the magnetic-MIP suspensions were separated by external neodymium magnetic bar and the supernatant was measured at a wavelength of $270 \mathrm{~nm}$.

For evaluating the selectivity, $5.0 \mathrm{mg}$ of magnetic-MIP were mixed with $1.0 \mathrm{~mL}$ of $1 \mathrm{mmol} \mathrm{L}^{-1}$ of different compounds including methyl parathion, malathion, 4-nitrophenol and phenol (263.2, 330.3; 139.1 and $94.1 \mathrm{mg} \mathrm{L}^{-1}$, respectively) with related structure, and shaken in a rotary shaker for $60 \mathrm{~min}$. After separation of the magnetic-MIP, the supernatant was measured at maximum absorption wavelength of each compound ( $\left.\lambda_{\max }=270,230,274,312\right)$ for methyl parathion, malathion, phenol and 4-nitrophenol, respectively. The distribution coefficient $\left(\mathrm{K}_{\mathrm{d}}, \mathrm{mL} \mathrm{g}^{-1}\right)$, selectivity $(\alpha)$, imprinting factor $(I)$ and the relative selectivity coefficient $(\beta)$ were calculated by the following equations (Clausen et al., 2014; Tarley et al., 2005).

$K_{d}=\frac{Q_{e}}{C_{e}}$

$\alpha=\frac{K_{d}(\text { methyl parathion })}{K_{d}(\text { interference })}$

$I=\frac{K_{d}(\text { magnetic }-M I P)}{K_{d}(\text { magnetic }-N I P)}$

$\beta=\frac{\alpha(\text { magnetic }- \text { MIPs })}{\alpha(\text { magnetic }- \text { NIPs })}$

The reusability study was performed by binding the magnetic-MIP $\left(5.0 \mathrm{mg}\right.$ ) with $1.0 \mathrm{~mL}$ of $26.3 \mathrm{mg} \mathrm{L}^{-1}$ of methyl parathion for $60 \mathrm{~min}$, followed by supernatant determination by UV spectrophotometry at $270 \mathrm{~nm}$, and subsequently washing with methanol:acetic acid for complete removal of the methyl parathion residues. This adsorption/ desorption cycle was repeated 6 times.

\subsection{Electrochemical sensing on m-GEC electrodes of methyl parathion by preconcentration on magnetic-MIP}

The direct electrochemical sensing of methyl parathion was achieved for the first time by preconcentration on the magnetic-MIP with further magnetic actuation on the surface of m-GEC electrode, as schematically shown in Fig. 1.

Firstly, the electrochemical characterization of methyl parathion on bare m-GEC electrodes $\mathrm{Ag} / \mathrm{AgCl} / \mathrm{KCl}$ (satd.) reference electrode were performed by cyclic voltammetry (CV). The optimization of the BR buffer ( $\mathrm{pH}=2,4,6$ and 8 ) was also performed by CV. Furthermore, the direct electrochemical detection of methyl parathion (without preconcentration on magnetic-MIP) was performed on bare m-GEC electrodes by square wave voltammetry (SWV) and using the following parameters: pulse amplitude $0.05 \mathrm{~V}$, step potential $0.01 \mathrm{~V}$ and frequency $10 \mathrm{~Hz}$. To achieve that, $100 \mu \mathrm{L}$ methyl parathion (ranging from
0.0 to $526.0 \mathrm{mg} \mathrm{L}^{-1}$ ) was spiked in $20.0 \mathrm{~mL}$ BR buffer $(\mathrm{pH}=2)$.

Finally, the detection of methyl parathion preconcentrated on magnetic-MIP by magnetic actuation on m-GEC electrode was performed. To achieve that, the magnetic-MIPs $\left(100 \mu \mathrm{L}\right.$ at $\left.5.0 \mathrm{mg} \mathrm{mL}^{-1}\right)$ was incubated with $100 \mu \mathrm{L}$ methyl parathion ranging from $6.610^{-3}$ to $2.6 \mathrm{mg} \mathrm{L}^{-1}$ in ethanol for $60 \mathrm{~min}$ with gently shaking at room temperature. The magnetic-MIP with the preconcentrated methyl parathion was then magneto-actuated on the surface of the m-GEC electrode for the electrochemical readout by SWV at step potential $0.01 \mathrm{~V}$, amplitude $0.05 \mathrm{~V}$, frequency $10 \mathrm{~Hz}$, and scan rate ranging from -0.2 to $-0.9 \mathrm{~V}$ vs. $\mathrm{Ag} / \mathrm{AgCl} / \mathrm{KCl}($ satd.) reference electrode. All experiments were performed in BR buffer ( $\mathrm{pH}=2$ ) as supporting electrolyte.

\subsection{Selectivity study}

As the chemical residues are usually found collectively in the environment, the study of the effect of related and/or similar compounds which can eventually interfere in the analysis must be addressed (Tan et al., 2010; Zhao et al., 2013; Xu et al., 2014; Xue et al., 2014). To achieve that, and under the same optimized conditions, $100 \mu \mathrm{L}$ of magnetic-MIP $(5.0 \mathrm{mg})$ was incubated with $100 \mu \mathrm{L}$ of $0.263 \mathrm{mg} \mathrm{L}^{-1}$ $\left(1.0 \mu \mathrm{mol} \mathrm{L}^{-1}\right)$ of methyl parathion in the presence of the same concentration $\left(1.0 \mu \mathrm{mol} \mathrm{L}^{-1}\right)$ of malathion, 4-nitrophenol and phenol (0.330, 0.139 and $0.094 \mathrm{mg} \mathrm{L}^{-1}$, respectively) and determined by SWV in BR buffer $(\mathrm{pH}=2)$.

\subsection{Electrochemical sensing of methyl parathion in fish samples}

Complex food samples as fish require very laborious and time consuming pretreatments for the accurate detection of pesticides by the traditional methods (Song et al., 2014). In this work, the magnetic-MIP was used as an alternative method for the extraction, clean up and preconcentration of methyl parathion from fish samples. To achieve that, muscles of tuna and cat fish purchased from local fish market retail stores in Barcelona (Spain), were homogenized by food blender and spiked with methyl parathion in concentrations of 0.025, 0.050 and $0.100 \mathrm{mg} \mathrm{Kg}^{-1}$. The spiked fish samples were then mixed with ethanol and stirred for $90 \mathrm{~min}$ for extraction of methyl parathion. The extract was then centrifuged and filtered. The filtrate $(100 \mu \mathrm{L})$ was mixed with $100 \mu \mathrm{L}$ of magnetic-MIP $\left(5.0 \mathrm{mg} \mathrm{mL}^{-1}\right)$ for the electrochemical determination.

\section{Results and discussion}

\subsection{Computer simulation for the rational design of the molecularly imprinted polymer for methyl parathion}

The rational design of the MIP with affinity towards methyl parathion was performed by the M062X/6-31G(d,p) level of theory in order to study not only the template and monomer-monomer interactions, but
A. Preconcentration of methyl parathion on magnetic-MIP

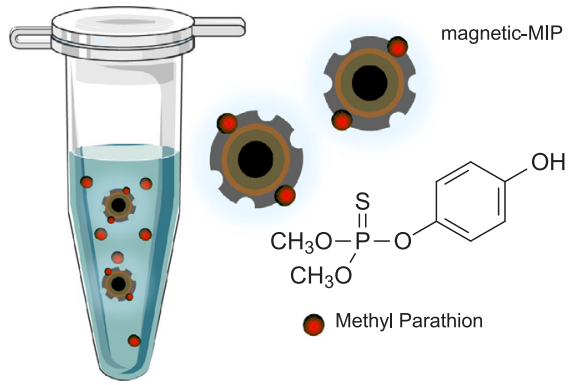

B. Magnetic actuation on magneto-electrodes

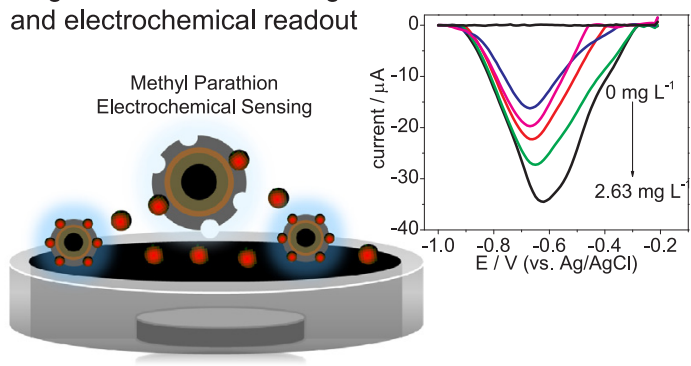

Fig. 1. Schematic procedure for the electrochemical sensing of methyl parathion preconcentrated on magnetic-MIP by magnetic actuation on m-GEC electrodes. 
also to assess the stoichiometry, noncovalent binding energies, solvation effects and thermodynamics properties such as binding energy. A detailed discussed of the theoretical studies are provided in Supp data. To summarize, among the 10 monomers studied in silico (Table S1, Supp. data), the methacrylic acid was considered to be the most suitable monomer, showing improved binding features towards methyl parathion. The methacrylic acid-methyl parathion complex shows a strong interaction due to hydrogen bonding, which presents a high directionality (Fig. S4, Supp. data). Furthermore, the use of toluene as solvent provides further stabilization of the complex due to the weakest interference on the interaction on methacrylic acid and methyl parathion complex (Fig. S5 and Table S2, Supp. data). Due to the high noncovalent binding energy and directionality provided by hydrogen binding, the synthesis was based on methacrylic acid as functional monomer and toluene as solvent. Although methacrylic acid was reported as monomer for methyl parathion (Zhao et al., 2013), no theoretical study was previously shown for the rational design of the MIP. Furthermore, the synthesis based on methacrylic acid as monomer for methyl parathion was performed on methanol and chloroform in the previous work (Xu et al., 2014; Zhao et al., 2013).

\subsection{Synthesis and characterization of the magnetic molecularly imprinted polymers for methyl parathion}

The detailed core-shell synthesis is described in Supp. data (Schemes S1-S3), and summarized in Fig. S2. First of all, the magnetic core of $\mathrm{Fe}_{3} \mathrm{O}_{4}$ nanoparticle was evaluated by selected-area electron diffraction (Fig. S6 and Table S3), and by X-ray diffraction (Fig. S7, Supp. data) to elucidate the crystal structure of the material. X-ray diffraction (XRD) of the $\mathrm{Fe}_{3} \mathrm{O}_{4}, \mathrm{Fe}_{3} \mathrm{O}_{4} @ \mathrm{SiO}_{2}$-TEOS, $\mathrm{Fe}_{3} \mathrm{O}_{4} @ \mathrm{SiO}_{2}-\mathrm{MPS}$ and $\mathrm{Fe}_{3} \mathrm{O}_{4} @$ MIP nanoparticles revealed six characteristic peaks located at $2 \theta$ of $30.36^{\circ}, 35.61^{\circ}, 43.47^{\circ}, 53.56^{\circ}, 57.10^{\circ}$ and $62.75^{\circ}$ corresponding to $\mathrm{Fe}_{3} \mathrm{O}_{4}$ in the material (Fig. S7). The diffractograms showed the planes $220,311,400,422,511$ and 440 corresponding to a pure cubic spinel crystal structure of $\mathrm{Fe}_{3} \mathrm{O}_{4}$ (Pan et al., 2014). The XRD pattern revealed that the highly crystalline nature of the magnetic nanoparticles remained unchanged during the synthesis of magnetic-MIP. Furthermore, after each step of the core-shell synthesis, the products were collected and studied by SEM, TEM and elemental analysis by Energy dispersive $\mathrm{X}$-ray spectroscopy, and the results are presented in Figs. S8 (Supp. data). As the layers are added to the outer part of the magnetic core of the nanoparticles, the size increased, being spherical in shape (Fig. S8). The average particle diameter ranges from $15 \mathrm{~nm}$ (SD 2) for the magnetite core of $\mathrm{Fe}_{3} \mathrm{O}_{4}$, to $77 \mathrm{~nm}$ (SD 18) for the first layer $\left(\mathrm{Fe}_{3} \mathrm{O}_{4} @ \mathrm{SiO}_{2}\right)$ and $179 \mathrm{~nm}$ (SD 23) for the second layer $\left(\mathrm{Fe}_{3} \mathrm{O}_{4} @ \mathrm{SiO}_{2}\right.$-MPS). For the
magnetic-MIP and magnetic-NIP it can be concluded that the size increases by TEM images, but it was not possible to determine the diameter on a representative number of entities due to the aggregation and the superposition of the different polymerization layers. The aspect of magnetic-MIP and magnetic-NIP are comparatively shown in Fig. 2. It was noticed the differences in porosity pattern of the magnetic-MIP (panel A) compared with the magnetic-NIP (panel B), which can be attributed to the cavities after methyl parathion extraction. Furthermore, as also confirmed by SEM (Fig. S9), the Fig. 2 reveals irregular spherical shape nanoparticles highly cross-linked among them. Moreover, the polymer includes many nanoparticles of magnetite in the structure, in agreement with the high magnetization of the material (Videos S1, Supp. data).

\subsection{Binding and selective adsorption study}

Different parameters involving the binding of methyl parathion on the magnetic-MIP were firstly studied by UV spectrophotometry (Fig. S11), including the study of the adsorption capacity $\left(Q, \mathrm{mg} \mathrm{g}^{-1}\right)$, the effect of adsorption time and the amount of magnetic-MIP on the adsorption capacity, the distribution coefficient $\left(K_{d}\right)$, selectivity $(\alpha)$, imprinting factor $(I)$ and the relative selectivity coefficient $(\beta)$ and the reusability of the magnetic-MIP as detailed in Supp. data.

The effect of binding time on the adsorption capacity of methyl parathion at low concentration range on magnetic-MIP is shown in Fig. S12, reaching the maximum $Q_{e}$ at $60 \mathrm{~min}$. This incubation time was thus selected for further studies. The influence of the amount of magnetic-MIP is presented in Fig. S13, showing, as expected, that the $Q_{e}$ value increases as the amount of magnetic-MIP increases up to $5.0 \mathrm{mg}$. The study of the kinetics adsorption for methyl parathion at different concentration (0.0-263.2 $\mathrm{mg} \mathrm{L}^{-1}$ ) on magnetic-MIP (and magnetic-NIP as a control) is shown in Fig. 3. The maximum adsorption capacity $\left(Q_{\max }\right)$ and the binding constant were thus estimated by a Langmuir model. $\left(\mathrm{R}^{2}=0.992\right)$, as detailed in Supp. data. The maximum adsorption capacity $\left(\mathrm{Q}_{\max }\right)$, and Langmuir affinity constant $\left(\mathrm{K}_{\mathrm{L}}\right)$ were calculated as the intercept and slope from the linearization, being the values $8.37 \mathrm{mg} \mathrm{g}^{-1}$, and $0.029 \mathrm{~L} \mathrm{mg}^{-1}$, respectively. The imprinting factor was found to be 3.99, (Table S4, Supp. data) higher than other molecularly imprinted polymer used for the determination of methyl parathion (Zhang et al., 2012).

The selectivity of the magnetic-MIP was comparatively study by using compounds with similar structure to methyl parathion, such as malathion, 4-nitrophenol, and phenol. The result of selectivity is shown in Table S4 and Fig. S14. The imprinting factor ( $I$ ) of magnetic-MIP for methyl parathion (3.99) was found to be higher than for the other

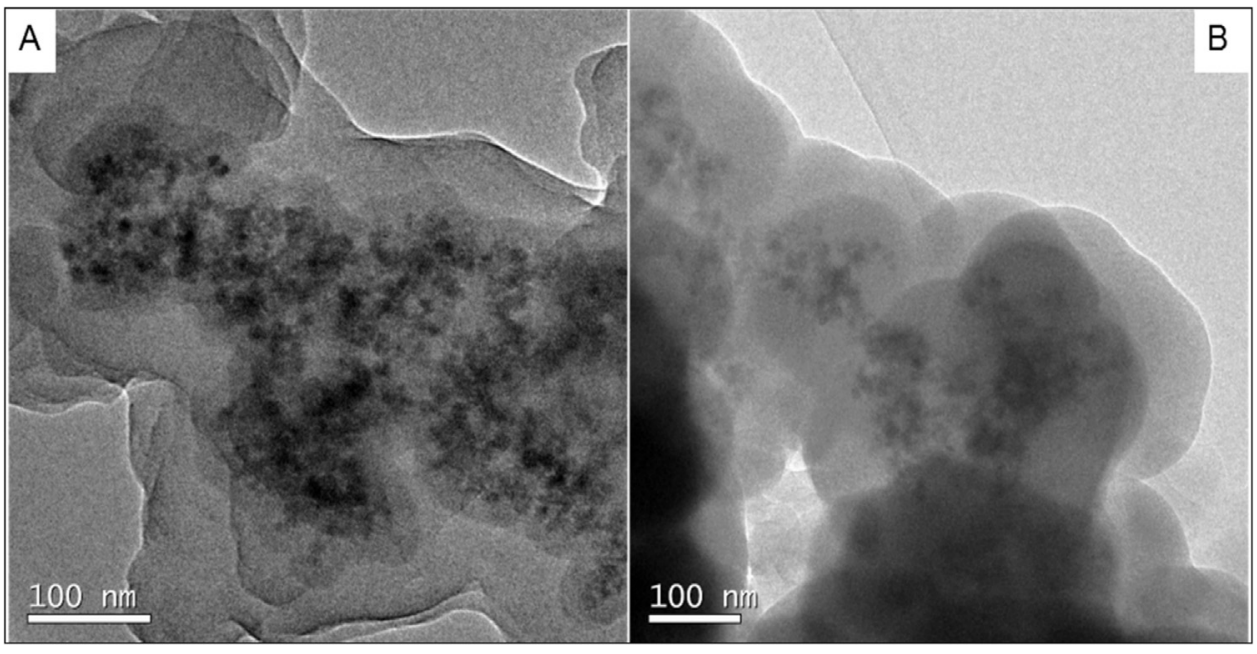

Fig. 2. Comparatively study at high resolution level of magnetic-MIP (A) and magnetic-NIP (B) by TEM operated at $200 \mathrm{kV}$. 


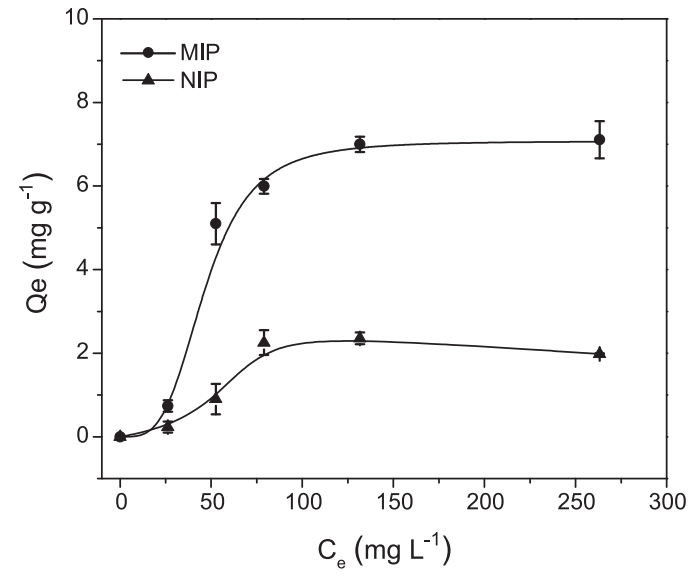

Fig. 3. Adsorption Langmuir isotherm of magnetic-MIP (and magnetic-NIP as a control) (5.0 mg) with different concentrations of methyl parathion $\left(0.0-263.2 \mathrm{mg} \mathrm{L}^{-1}\right)$ in ethanol. The binding time was $60 \mathrm{~min}$. Error bar shows the standard deviation $(\mathrm{n}=3)$.

substances (2.58, 1.28, 1.29 for malathion, 4-nitrophenol and phenol, respectively), confirming the specific binding sites similar in shape and size to methyl parathion. This fact is more evident in case of phenol and 4-nitrophenol, as the binding capacity of both magnetic-MIP and magnetic-NIP were similar that can be attributed to non-specific binding. For malathion, the higher imprinting factor may attributed to the similarity in the structure between methyl parathion and malathion.

Reusability is of a great cost benefit and it is considered a key factor in the application of solid phase extraction materials (Gao et al., 2011). Furthermore, reusability is technically more easily performed in magnetic-MIP than in conventional MIPs by magnetic actuation. In the reusability experiment, magnetic-MIP was used for the adsorption of methyl parathion in six consecutive adsorption/desorption cycles. The adsorption capacity for each cycle is shown in Fig. S15. The results were statistically analyzed by Student's t-test of the linear regression slope, with a significance of 0.05 . The linear regression model (Fig. S15) shows a non significant decrease of about $2.7 \%$ in the binding capacity after six adsorption/desorption cycles. This non-significant decrease in the binding capacity may be attributed to loss of some binding sites due to repeated washing (Gao et al., 2014). From this result, it can be concluded that there is no significant tendency to decrease in the binding capacity of the magnetic-MIPs after six adsorption/desorption cycles, suggesting that the magnetic MIP can be used even during more cycles. This indicates that magnetic-MIP shows an outstanding reusability and can be washed and reused at least during six cycles without a significant decrease in the binding capacity.

\subsection{Electrochemical sensing on m-GEC electrodes of methyl parathion by preconcentration on magnetic-MIP}

Firstly, the electrochemical characterization of methyl parathion on bare m-GEC electrodes (against $\mathrm{Ag} / \mathrm{AgCl}$ sat) were performed by $\mathrm{CV}$, as detailed shown in Fig. S16. A sharp cathodic current peak was observed in the first cycle at $-0.65 \mathrm{~V}$, which corresponded to the reduction of the nitro group to hydroxylamine group through a four electron process. The optimization of the $\mathrm{pH}$ was also performed. Improved electrochemical performance for the detection was achieved at $\mathrm{pH} 2$ for the $\mathrm{BR}$ buffer (Fig. S17). Furthermore, the direct electrochemical detection of methyl parathion (without preconcentration on magnetic-MIP) was performed on bare m-GEC electrodes by SWV by spiking $100 \mu \mathrm{L}$ methyl parathion (ranging from 0.0 to $526.0 \mathrm{mg} \mathrm{L}^{-1}$ ) in $20.0 \mathrm{~mL}$ of BR buffer $(\mathrm{pH}=2)$ (Fig. S21, panel B). The cathodic peak current was fitted by a linear regression $\left(R^{2}=0.9472\right)$ (Fig. S21, panel B). The limit of detection (LOD) for the detection of methyl parathion on the bare m-GEC electrode was thus calculated, by processing the negative control samples (BR buffer only) $(n=8)$, obtaining a mean value of $0.166 \mu \mathrm{A}$ / SD 0.028 . The cut-off value was then determined with a one-tailed $t$-test at a $95 \%$ confidence level, giving values of $0.219 \mu \mathrm{A}$. This value was interpolated and the LOD was found to be $16.35 \mathrm{mg} \mathrm{L}^{-1}$, much higher than the permissible limit for methyl parathion in food (0.05-0.20 $\mathrm{mg} \mathrm{Kg}^{-1}$ ) (FAO/WHO, 1986). In order to improve the limit of detection, $100 \mu \mathrm{L}$ of methyl parathion, ranging from 0.0 to 2.6 $\mathrm{mg} \mathrm{L}^{-1}$ was preconcentrated on the magnetic-MIP for $60 \mathrm{~min}$ and then the magnetic-MIP was captured on the surface of m-GEC for direct electrochemical determination of methyl parathion by SWV. The results are shown in Fig. 4, panel A. The maximal cathodic peak current was fitted by a nonlinear regression (Two site binding) $\left(\mathrm{R}^{2}=0.9984\right)$ (Fig. 4, panel B). The LOD for the detection of methyl parathion preconcentrated on the magnetic-MIP was thus calculated, by processing the negative control samples $(n=4)$, obtaining a mean value of $0.02435 \mu \mathrm{A} / \mathrm{SD} 0.00099 \mu \mathrm{A}$. The cut-off value was then determined
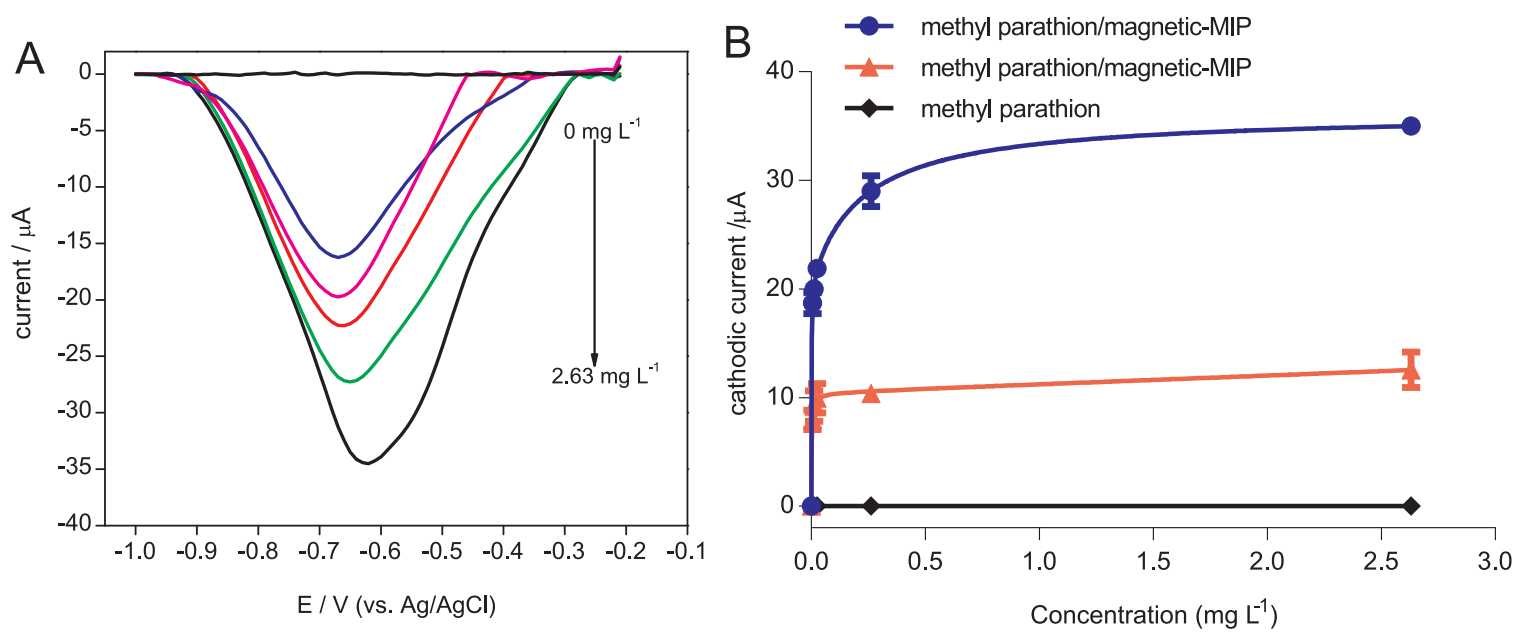

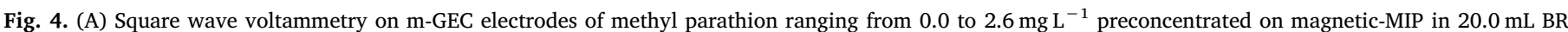

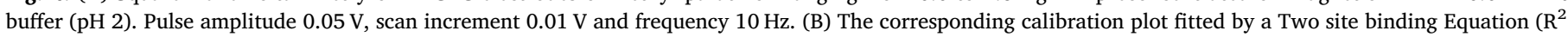

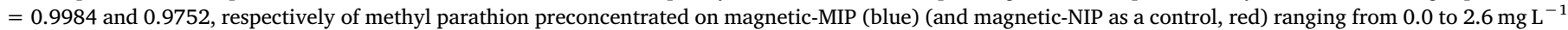

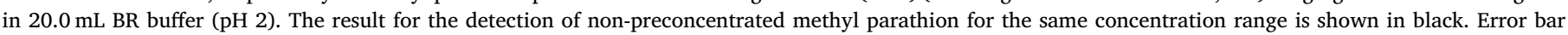

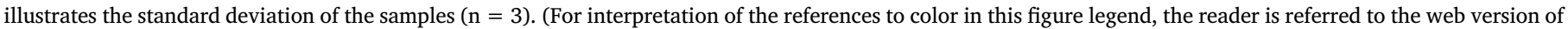
this article.). 


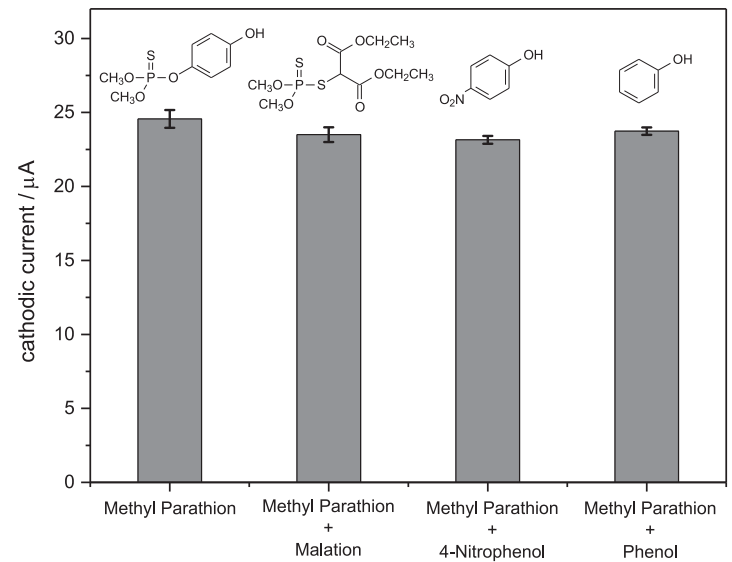

Fig. 5. Selectivity study of magnetic-MIP for methyl parathion $\left(0.263 \mathrm{mg} \mathrm{L}^{-1}\right)$ in the presence of $1.0 \mu \mathrm{mol} \mathrm{L}^{-1}$ of related compounds: malathion, 4-nitrophenol and phenol and determined ( $0.330,0.139$ and $0.094 \mathrm{mg} \mathrm{L}^{-1}$, respectively) by SWV at m-GEC electrode in $20.0 \mathrm{~mL}$ of BR buffer (pH 2). Pulse amplitude $0.05 \mathrm{~V}$, scan increment $0.01 \mathrm{~V}$ and frequency $10 \mathrm{~Hz}$. Error bar shows the standard deviation $(\mathrm{n}=3)$.

with a one-tailed $t$-test at a $95 \%$ confidence level, giving a value of $0.02669 \mu \mathrm{A}$. This value was interpolated and the LOD was found to be as low as $1.22 \times 10^{-6} \mathrm{mg} \mathrm{L}^{-1}$, much lower than the permissible limit in food. Regarding the previously reported work using direct electrochemical detection on molecularly imprinted-modified sensors, methyl parathion was determined in river water with a detection limit of $1.0 \times 10^{-2} \mathrm{mg} \mathrm{L}^{-1}$ (Xue et al., 2014). Moreover, limits of detection of $2.3 \times 10^{-3}, 1.6 \times 10^{-3}$ and $1.8 \times 10^{-2} \mathrm{mg} \mathrm{L}^{-1}$ were detected in pear juice, cabbage and apple peel and cucumber samples, respectively (Tan et al., 2010; Zhao et al., 2013; Zhang et al., 2012). Comparing to the aforementioned studies, the LOD of the current work was found to be much lower. This may be attributed to the ability of magnetic-MIP to bind and concentrate methyl parathion on the surface of m-GEC. Fig. 4, panel B also shown the direct cathodic current signals for methyl parathion determined by the bare m-GEC electrode (without preconcentration) at the same concentration range, highlighting the outstanding capacity of the magnetic-MIP to preconcentrated methyl parathion and thus to improve the LOD.

\subsection{Selectivity study}

Fig. 5 shows the selectivity of the magnetic-MIP for methyl parathion in the presence of different related compounds with similar structure to methyl parathion including malathion, 4-nitrophenol and phenol. As shown in Fig. 5, there are no significant differences in the cathodic current when the interferences are present in the sample. The results of the statistical analysis performed by on way ANOVA $(p>0.01)$ revealed that there are no significant differences between the cathodic current obtained by methyl parathion preconcentrated on magnetic-MIP alone and in the presence of other foreign substance. This good selectivity may be attributed to the specific binding sites of methyl parathion on magnetic-MIP.

\subsection{Electrochemical sensing of methyl parathion in fish samples}

Table 1 shows the recovery values for methyl parathion in fish
Table 1

Recovery study for methyl parathion in spiked fish samples $(n=3)$.

\begin{tabular}{lllll}
\hline Fish type & $\begin{array}{l}\text { Methyl parathion } \\
\text { spiked }\left(\mathrm{mg} \mathrm{Kg}^{-1}\right)\end{array}$ & $\begin{array}{l}\text { Methyl parathion } \\
\text { found }\left(\mathrm{mg} \mathrm{Kg}^{-1}\right) \\
\text { mean/SD }\end{array}$ & RSD \% & Recovery \% \\
\hline \multirow{2}{*}{ Tuna } & 0.0000 & 0.0000 & 0.0 & 0.0 \\
& 0.0250 & $0.0236 / 0.0002$ & 0.7 & 94.7 \\
& 0.0500 & $0.047 / 0.002$ & 4.0 & 94.6 \\
Cat fish & 0.1000 & $0.092 / 0.004$ & 3.9 & 92.0 \\
& 0.0000 & 0.0000 & 0.0 & 0.0 \\
& 0.0250 & $0.0230 / 0.0008$ & 3.3 & 92.1 \\
& 0.0500 & $0.047 / 0.002$ & 4.0 & 94.6 \\
& 0.1000 & $0.089 / 0.002$ & 2.4 & 89.4 \\
\hline
\end{tabular}

samples from local retail markets (tuna and cat fish) performed by preconcentration on the magnetic-MIPs, magnetic actuation on m-GEC electrodes, and electrochemical sensing by SWV. The average recoveries and RSD \% ranging from $89.4 \%$ to $94.7 \%$. This satisfactory recovery rate proves that the synthesized magnetic-MIP keeps the affinity towards the template even in complex samples, and suggesting that it can be used for the rapid determination of methyl parathion residues in complex food samples.

\section{Conclusion}

This work addresses the development of a new approach the direct electrochemical detection of methyl parathion based on the preconcentration of the pollutant by using magnetic-MIP integrated on a magneto-actuated electrode. The magnetic-MIP showed a high adsorption capacity, selectivity, reusability and outstanding magnetic properties. Due to the analytical performance, this approach offers an exciting alternative to the current methods for detection of methyl parathion, including assay time, simplicity, long-term storage, LOD (much lower than the permissible limit in food), low cost and animalfree production of reagents. The preconcentration of methyl parathion from fish samples also showed outstanding recovery values, demonstrating the ability of this material to preconcentrate the pollutant from complex food samples. This simple and rapid method will open the way to incorporate this material in different magneto-actuated devices in the field of food safety and environmental monitoring with no need to extensive sample pretreatment and sophisticated instruments. Future work will be focused on challenging the test in different contaminated samples by comparing with the gold standard method in collaboration with a reference food safety laboratory.

\section{Acknowledgment}

Financial support from the Ministry of Economy and Competitiveness (MINECO), Madrid (Grants BIO2013-41242-R and BIO2016-75751-R) are gratefully acknowledged. Egyptian Government is gratefully acknowledged for her assistance. Department of Chemistry of the Instituto Tecnológico de Aeronáutica and Conselho Nacional de Desenvolvimento Científico e Tecnológico, Ministry of Science, Technology, Innovation and Communications of Brazil (Grant 233595/ 2014-7) are gratefully acknowledged.Conflict of interests

The authors have no conflict of interests.

\section{Appendix A. Supporting information}

Supplementary data associated with this article can be found in the online version at doi:10.1016/j.bios.2018.06.052. 


\section{References}

Ben Aissa, A., Jara, J.J., Sebastián, R.M., Vallribera, A., Campoy, S., Pividori, M.I., 2017. Biosens. Bioelectron. 88, 265-272.

Brandão, D., Liébana, S., Pividori, M.I., 2015. New Biotechnol. 32, 511-520. Carinelli, S., Martí, M., Alegret, S., Pividori, M.I., 2015. New Biotechnol. 32, 521-532. Clausen, D.N., Visentainer, J.V., Tarley, C.R.T., 2014. Analyst 139, 5021-5027.

Duan, H., Li, L., Wang, X., Wang, Y., Li, J., Luo, C., 2015. Spectrochim. Acta A 139, 374-379.

FAO/WHO, 1986. Codex Maximum Limits for Pesticide Residues, 3rd ed. Codex Alimentarius Commission, Food and Agriculture Organization of the United Nations (CAC/Vol XIII), Rome.

Gao, R.X., Kong, X., Wang, X., He, X.W., Chen, L.X., Zhang, Y.K., 2011. J. Mater. Chem. $21,17863-17871$.

Gao, R., Hao, Y., Zhao, S., Zhang, L., Cui, X., Liu, D., Tang, Y., Zheng, Y., 2014. RSC Adv. 4, 56798-56808.

Huang, G., Ouyang, J., Baeyens, W.R.G., Yang, Y., Tao, C., 2002. Anal. Chim. Acta 474, 21-29.

Lagaly, G., 2001. Appl. Clay Sci. 18, 205-209.

Liu, G., Lin, Y., 2005. Anal. Chem. 77, 5894-5901.

Ma, R.T., Shi, Y.P., 2015. Talanta 34, 650-656.

Moura, S.L., Martinez Fajardo, L., Cunha, L.A., Sotomayor, M.D.P.T., Machado, F.B.C., Ferrão, L.F.A., Pividori, M.I., 2018. Biosens. Bioelectron. 107, 203-210.

Pan, S.D., Shen, H.Y., Zhou, L.X., Chen, H.X., Zaho, Y.G., Cai, M.Q., Jin, M.C., 2014. J.
Mater. Chem. A 2, 15345-15356.

Pividori, M.I., Alegret, S., 2005. Anal. Lett. 38, 2541-2565.

Rembaum, A., Yen, R.C.K., Kempner, D.H., Ugelstad, J., 1982. J. Immunol. Methods 52, $341-351$.

Sanz, C.P., Halko, R., Ferrera, Z.S., 2004. Anal. Chim. Acta 524, 265-270.

Silva, D., Cortez, C.M., Cunha-Bastos, J., Louro, S.R.W., 2004. Toxicol. Lett. 147, 53-61. Shanker, A., Sood, C., Kumar, V., Ravindranath, S.D., 2001. Pest Manag. Sci. 57, 458-462. Song, X., Xu, S., Chen, L., Wei, Y., Xiong, H., 2014. J. Appl. Polym. Sci. 131, 40766-40783.

Tarley, C.R.T., Sotomayor, M.D.P.T., Kubota, L.T., 2005. Quím. Nova 28, 1076-1086.

Tan, X., Li, B., Liew, K.Y., Li, C., 2010. Biosens. Bioelectron. 26, 868-871.

Tomkins, B.A., Ilgner, R.H., 2002. J. Chromatogr. A 972, 183-194.

Vlatakis, G., Andersson, L.I., Müller, R., Mosbach, K., 1993. Nature 361, 645-647.

Vogt, R., Bennett, D., Cassady, D., Frost, J., Ritz, B., Picciotto, H.I., 2012. Environ. Health $11,83-96$.

Xu, S., Guo, C., Li, Y., Yu, Z., Wei, C., Tang, Y., 2014. J. Hazard. Mater. 264, 34-41.

Xue, X., Wei, Q., Wu, D., Li, H., Zhang, Y., Feng, R., Du, B., 2014. Electrochim. Acta 116, $366-371$.

Zacco, E., Pividori, M.I., Alegret, S., Galve, R., Marco, M.P., 2006. Anal. Chem. 78, 1789-1798.

Zhao, L., Zhao, F., Zeng, B., 2013. Sens. Actuator B Chem. 176, 818-824.

Zhang, D., Yu, D., Zhao, W., Yang, Q., Kajiura, H., Li, Y., Zhou, T., Shi, G., 2012. Analyst $137,2629-2636$ 